\title{
Simulação Baseada em Multiagentes como Ferramenta em Estudos Interdisciplinares
}

\author{
Diana F. Adamatti \\ Centro de Ciências Computacionais \\ Universidade Federal do Rio Grande (FURG) - Brasil \\ dianaadamatti@furg.br
}

\section{Resumo}

Este artigo apresenta estudos de casos com alunos de graduação e pós-graduação que foram introduzidos a área de Agentes e Simulação Baseada em Multiagentes, desenvolvendo experimentos com essas técnicas computacionais, visando desenvolver habilidades junto a fenômenos interdisciplinares.

Palavras-chave: simulação multiagentes, interdisciplinariedade

\begin{abstract}
This paper presents some case studies with undergraduate and graduate students that they were introduced in agents and multi-agent-based simulation areas, basing in experiments with these computational techniques. The main goal was to develop skills in interdisciplinary phenomenas.
\end{abstract}

Keywords: multi-agent-based simulation, interdisciplinary

\section{Introdução}

Os Sistemas Multiagentes (SMA) estudam o comportamento de um conjunto de agentes autônomos, eventualmente com características diferentes, evoluindo em um ambiente comum. Estes agentes podem interagir uns com os outros, com o objetivo de realizar suas tarefas de modo cooperativo, compartilhando informações, evitando conflitos e coordenando a execução de atividades (Alvares e Sichman, 1997). Adicionalmente, a utilização da simulação como elemento auxiliar na tomada de decisão humana tende a ser muito eficaz, pois seu emprego permite o exame de detalhes específicos com grande precisão (Strack, 1984). Da integração das tecnologias de agentes e de simulação, surgiu a área de Simulação Baseada em Multiagentes (MultiAgent-Based Simulation (MABS)), que é especialmente útil em domínios em que a interdisciplinaridade se faz presente (Gilbert e Troitzsch, 1999).

Segundo Fazenda (1994, a interdisciplinaridade é uma linha de pesquisa dos tempos modernos, que surgiu na França nos anos 60, com objetivo de sintonizar o ensino a grandes questões de ordem social, política e econômica e científica, que não poderiam ser resolvidos por uma única disciplina ou área do saber. A interdisciplinaridade ganhou força nas instituições de ensino, seja a nível básico, médio ou superior, principalmente no discurso dos professores. Contudo, estudos têm revelado que a interdisciplinaridade ainda é pouco conhecida e posta em prática (Carlos, 2011).

A área de Simulação Baseada em Agentes tem grande atividade de pesquisa na pósgraduação, inclusive contando, em diversos programas, com disciplinas específicas. 
Contudo, a utilização dessa técnica com alunos de graduação, em cursos da área de computação, ainda é restrita. Um fato que pode explicar esse processo é que, em disciplinas da graduação ligadas a Inteligência Artificial (IA), as ementas apresentam conteúdos de grandes áreas da IA, como Redes Neurais, Algoritmos Genéticos, Lógica Fuzzy, entre outros. Desta forma, o estudo mais aprofundado de Agentes e Simulação Baseada em Multiagentes não pode ser realizado.

Este artigo apresenta estudos de caso com alunos de graduação e pós-graduação que foram introduzidos a área de Agentes e Simulação Baseada em Agentes e realizaram experimentos de simulação em dois ambientes. Primeiro em um ambiente bastante simples de utilizar, que trabalha com uma formação de regras em português estruturado, denominado SIMULA (Frozza, 1997). Em seguida, em um ambiente mais complexo (relacionado a procedimentos possíveis) e completo, denominado NetLogo (NetLogo, 2011). O principal objetivo destes estudos de caso foi o de verificar se a utilização de Simulação Baseada em Multiagentes pode ser utilizada como ferramenta para estudos interdisciplinares e se os alunos tomaram consciência das atividades que estavam realizando.

O artigo é divido em 4 seções. Na seção 2 são apresentados os conceitos de agentes, simulação e simulação baseada em multiagentes. A seção 3 apresenta os estudos de caso realizados junto aos alunos e na seção 4 estão as conclusões encontradas.

\section{Simulação Baseada em Multiagentes}

Modelar um fenômeno sob a perspectiva de um SMApode ser realizado a partir das seguintes etapas (Frozza, 1997):

- Decompor o fenômeno em um conjunto de elementos autônomos;

- Modelar cada um dos elementos como um agente, definindo seu conhecimento, funções, comportamento e modos de interação;

- Definir o ambiente dos agentes;

- Definir quais agentes possuem a capacidade de ação e comunicação.

Já os métodos de simulação são empregados com grande sucesso como elemento auxiliar na tomada de decisões, principalmente no planejamento a médio e longo prazo e em situações que envolvem custos e riscos elevados. Os modelos de simulação são muito eficazes e versáteis no estudo dos mais diferentes problemas. Seu emprego permite o exame de detalhes específicos com grande precisão (Rebonatto, 1999).

Assim, a simulação baseada em computador é uma das ferramentas mais poderosas disponíveis nos dias de hoje para projetar, planejar, controlar e avaliar novas alternativas e/ou mudanças de estratégias em sistemas no mundo real. Sua utilização significa construir programas de computador (software) que 'representem' o sistema do mundo real em questão e 'imitem' seu funcionamento (Gilbert e Troitzsch, 1999; Rebonatto, 1999). A simulação pode ser dividida em três etapas (Strack, 1984):

- Etapa da Modelagem: construir o modelo do fenômeno a ser estudado;

- Etapa de Experimento: aplicar variações sobre o modelo construído, alterando parâmetros que influam no processo de resolução; 
- Etapa de Validação: comparar dados experimentais obtidos com o modelo e a realidade, permitindo a análise dos resultados obtidos.

Pela Figura 1, pode-se verificar as etapas de um processo de simulação. A partir da realidade, é realizada uma modelagem do problema desejado e é construído um modelo. Para se construir este modelo, têm-se como base uma teoria. Com o modelo, realiza-se a simulação e depois a avaliação do mesmo. Para a avaliação, utilizam-se os resultados obtidos pelo modelo e as observações da realidade (Drogoul e Ferber, 1992).

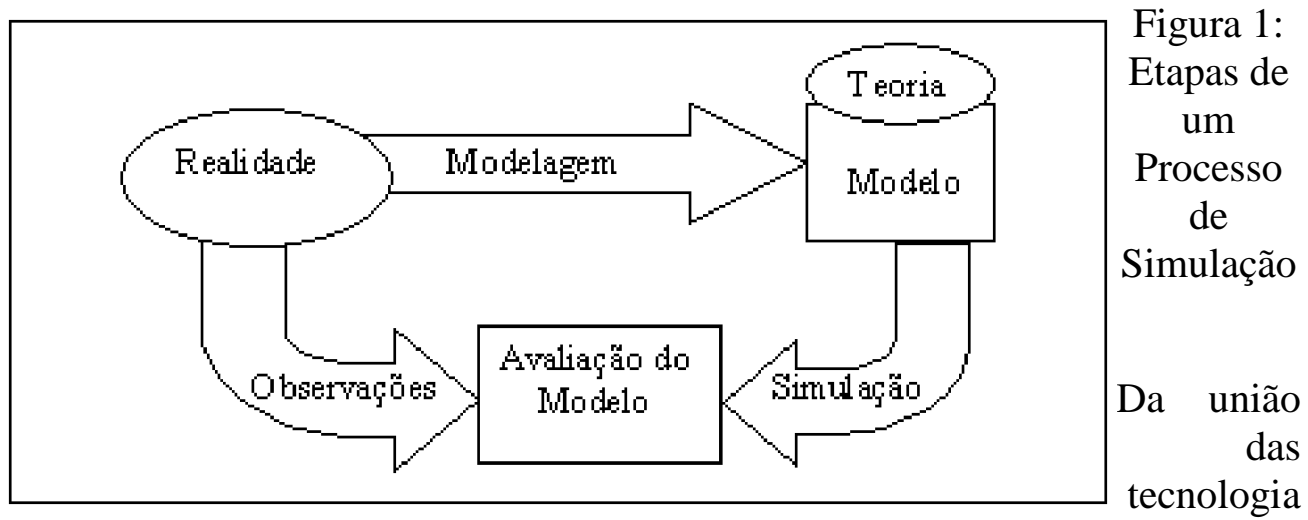

s de Simulação e Sistemas Multiagentes, surge a área de Simulação Baseada em Multiagentes (Multi-Agent-Based Simulatin (MABS)). Muitas aplicações em SMAs são desenvolvidas para simular alguma situação da realidade. Para tal, o fenômeno real é decomposto em um conjunto de elementos e em suas interações. Cada elemento é modelado como um agente e o modelo geral é o resultado das interações entre estes agentes.

Segundo Drogoul e Ferber (1992), os objetivos de MABS são:

- Testar hipóteses sobre a emergência de comportamentos no nível macro a partir de interações no nível micro;

- Construir teorias que contribuam para o entendimento de fenômenos, etológicos, sociológicos ou psico-sociais, que relacionam comportamentos a estruturas;

- Integrar teorias parciais de diferentes disciplinas (sociologia, psicologia cognitiva, etologia) numa mesma estrutura.

Alguns aspectos importantes em MABS são (Drogoul e Ferber, 1992):

- É uma técnica bastante adequada para situações onde existem muitos indivíduos, com comportamentos diversos e complexos;

- Pode-se levar em conta tanto aspectos quantitativos como aspectos qualitativos;

- Programam-se os comportamentos primitivos de cada indivíduo e o comportamento global que emerge das interações entre os indivíduos é analisado, mas não pré-programado de modo direto.

\section{Estudos de Caso Realizados}

Os estudos de caso foram realizados com alunos da Universidade Federal do Rio Grande (FURG), com alunos de graduação (Disciplina de Sistemas Inteligentes do 4o. ano do curso de Engenharia de Computação) e com alunos de pós-graduação (Disciplina 
de Simulação Social do Curso de Pós-Graduação em Modelagem Computacional).

$\mathrm{Na}$ disciplina da graduação, que é realizada em sistema de colegiado, em cada bimestre um professor ministra a sua área de pesquisa. No quarto bimestre, os alunos estudaram agentes, envolvendo comunicação, organização, tomada de decisão e simulação. Na disciplina de pós-graduação, os alunos estudaram simulação e modelos de simulação (micro, macro, dinâmica e baseada em multiagentes).

Para as duas disciplinas, foram exigidos dois trabalhos de simulação baseada em multiagentes: um utilizando o ambiente SIMULA e outro o ambiente NetLogo.

A descrição proposta para os trabalhos foi a seguinte:

1. Escolha um problema a ser modelado;

2. Especifique os agentes e regras do problema;

3. Especifique o ambiente do problema;

4. Implemente nos ambientes de simulação baseado em agentes SIMULA e NetLogo;

5. Elabore um relatório apresentando a evolução dos agentes durante a execução (um para cada ambiente).

A idéia foi que a descrição dos trabalhos fosse o mais genérica possível, dando aos estudantes a oportunidade de escolha de problemas em diversas áreas de conhecimento.

As etapas 1, 2, 3 e 4 da descrição dos trabalhos estão diretamente relacionadas com as etapas necessárias para modelar um fenômeno sob a perspectiva de um SMA, descrito por Frozza (1997).

A solicitação de um relatório com a evolução dos agentes (etapa 5) foi a forma encontrada para que os alunos percebessem como comportamentos primitivos de cada agente podem influenciar no comportamento global, mesmo que não implementado de forma direta.

Os trabalhos propostos estão diretamente relacionados ao objetivo de "teste" de hipóteses sobre a emergência de comportamentos, citado por Drogoul e Ferber (1992), visto que os alunos não construíriam teorias para o entendimento de fenômenos.

\subsection{Trabalhos Implementados}

Os trabalhos foram realizados individualmente ou em duplas. As áreas de conhecimento escolhidas pelos alunos foram as mais diversas, como biologia, economia, fisiologia e jogos. Não havia exigência que o segundo trabalho, no ambiente NetLogo, fosse igual ao desenvolvido no primeiro trabalho, no ambiente SIMULA. Contudo, diversos alunos preferiram desenvolver trabalhos iguais nos dois ambientes, pois não há a necessidade de conhecer um novo domínio do problema para a segunda implementação.

\subsubsection{Exemplos no Ambiente SIMULA}

As Figuras 2 e 3 apresentam alguns dos trabalhos desenvolvidos pelos alunos no ambiente SIMULA.

A Figura 2 apresenta como os alunos implementaram o conhecido jogo Pacman. Os alunos definiram os agentes Pac, Bola, Fantasma e Muro, e regras de movimentação, 
onde o Pac (rosto "smiles" na Figura 2) procura as Bolas (círculos pequenos em amarelo) e os Fantasmas (em verde) procuram o Pac. O agente Muro é um objeto estático, que foi utilizado para montar o ambiente do tipo labirinto (quadrados cinza). $\mathrm{O}$ objetivo da simulação é que o Pac "coma" todas as Bolas e não seja eliminado pelos Fantasmas.

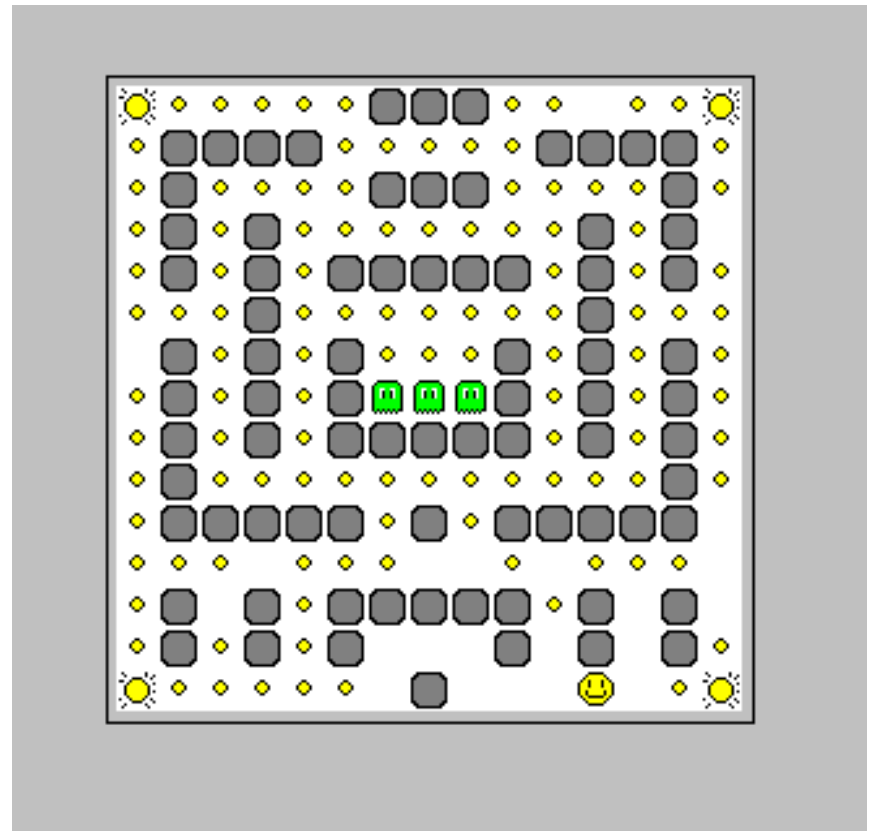

Exemplo de simulação no SIMULA: Pacman

Figura 2:

A Figura 3 apresenta a implementação do Funcionamento do Sistema Imunológico Humano quando atacado por um vírus. Os alunos definiram os agentes CélulaBoa, CélulaInfectada, Vírus e Anticorpo. A simulação inicia com uma quantidade grande de CélulasBoas (círculos com contorno preto na Figura3), uma quantidade baixa de Vírus (em verde) e uma quantidade muito baixa de Anticorpos (em azul). Os Vírus, quando encontram CélulasBoas, as atacam e estas tornam-se CélulasInfectadas (círculos com contorno em vermelho). Contudo, a prioridade de um Vírus é fugir dos Anticorpos. Os Anticorpos, quando percebem que o número de CélulasInfectadas cresce, se reproduzem e tentam matar os Vírus. Os Anticorpos tentam recurperar as CélulasInfectadas, para reestabelecer a estabilidade do sistema, ou seja, acabar com os Vírus.

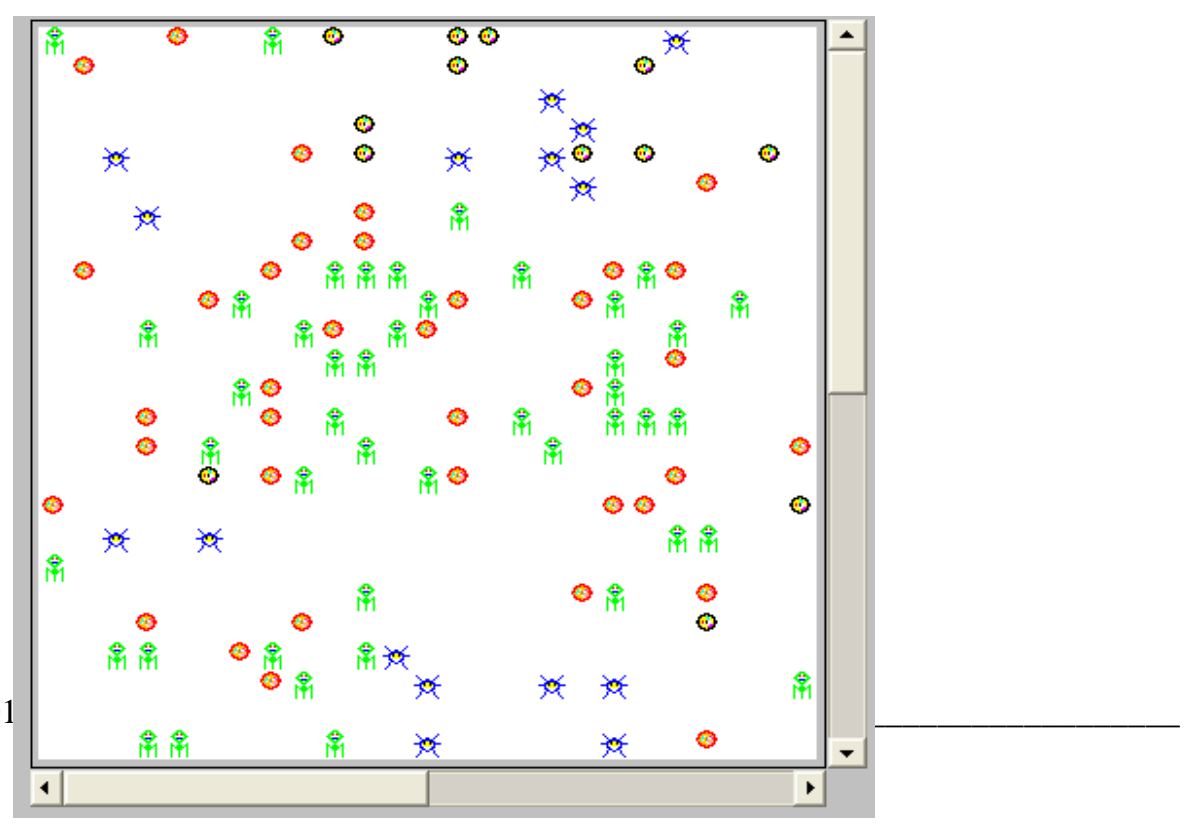


Figura 3: Exemplo de simulação no SIMULA: Funcionamento do Sistema Imunológico

Além dos dois exemplos apresentados acima, outros trabalhos muito interessantes foram implementados, como:

- Simulação da difusão da Doença da Dengue, onde foi apresentado como os mosquitos se reproduzem, mostrando as etapas de ovo e larva, e atacam as pessoas, infectando-as;

- Simulação de uma situação de guerra entre dois exércitos, onde estratégias diferentes foram criadas, e dependendo da configuração de cada exército, uma delas pode ser melhor que a outra;

- Simulação do jogo do Detetive, onde o Assassino pode estar disfarçado no meio das pessoas, e não ser descoberto.

\subsubsection{Exemplos no Ambiente NetLogo}

As Figuras 4 e 5 apresentam alguns dos trabalhos desenvolvidos pelos alunos no ambiente NetLogo.

A Figura 4 apresenta como os alunos implementaram a forma que o vírus H1N5 (gripe aviária) se alastra. A contaminação inicia em aves silvestres previamente contaminadas que transmitem o vírus através do contato, das fezes, ou ainda do solo ou água no qual estes excrementos foram postados. Devido sua alta concentração em um curto espaço, essa gripe se alastra muito rapidamente entre as aves domésticas, podendo chegar aos seres humanos. As pessoas adquirem o vírus de forma semelhante, ao manipularem aves contaminadas pela gripe, entrando em contato com seu sangue ou fezes. Normalmente decorrente de seu preparo para cozimento, onde há a necessidade de arrancar as penas. A transmissão entre seres humanos é mais rara mas ainda assim preocupante.

Os alunos definiram os agentes Agricultor, Ave Silvestre, Ave Doméstica e Pessoa. A simulação inicia com o número desejado de todos os agentes (boxes em azul no lado esquerdo da Figura 4), visto que o ambiente permite a criação de interface gráfica personalizada de forma facilitada
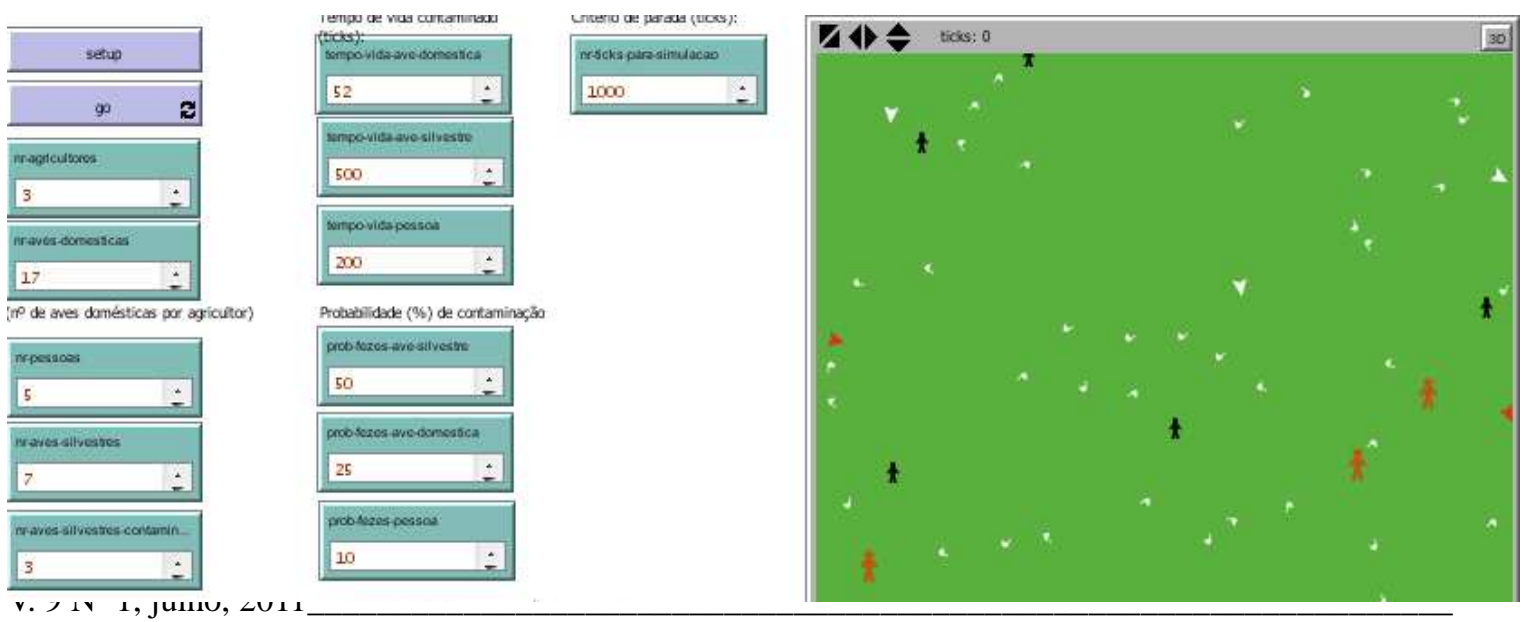
Figura 4: Exemplo de simulação no NetLogo: Funcionamento da Contaminação do Vírus H1N5

A Figura 5 apresenta como os alunos implementaram um ecossistema composto por microalgas, luz e nutrientes. As microalgas se movimentam randomicamente, bem como a luz e nutrientes são renovados da mesma maneira. Quando uma microalga encontra luz ou nutriente, ela absorve energia, para sobreviver. Os parâmetros luz e nutrientes podem ser setados com diferentes valores via interface, gerando ou um ambiente hostil ou mais agradável as microalgas. Os alunos definiram três agentes: Microalgas, Luz e Nutrientes. O ambiente também é dividido em duas partes: o mar profundo (em azul escuro), onde a luz não consegue penetrar de forma completa e devido ao frio as microalgas gastam mais energia para continuar vivas; e o mar superficial (em azul claro), onde a luz penetra de forma completa e tem-se mais nutrientes. Pela Figura 5 pode-se perceber que o trabalho também apresenta gráficos com a evolução da população em tempo de simulação. Esta é outra vantagem da utilização do NetLogo.

Além desses dois exemplos apresentados, os alunos também implementaram outros trabalhos, como:

- Simulação do ciclo de vida do sapo, de ovo para lavra, depois para gerino e finalmente a sapo;

- Simulação de uma situação de guerra entre quatro países, onde estratégias diferentes foram criadas, baseadas em força e armas disponíveis;

- Simulação de engenharia de segurança, onde verifica-se a evacuação de espaço em determinado período de tempo.

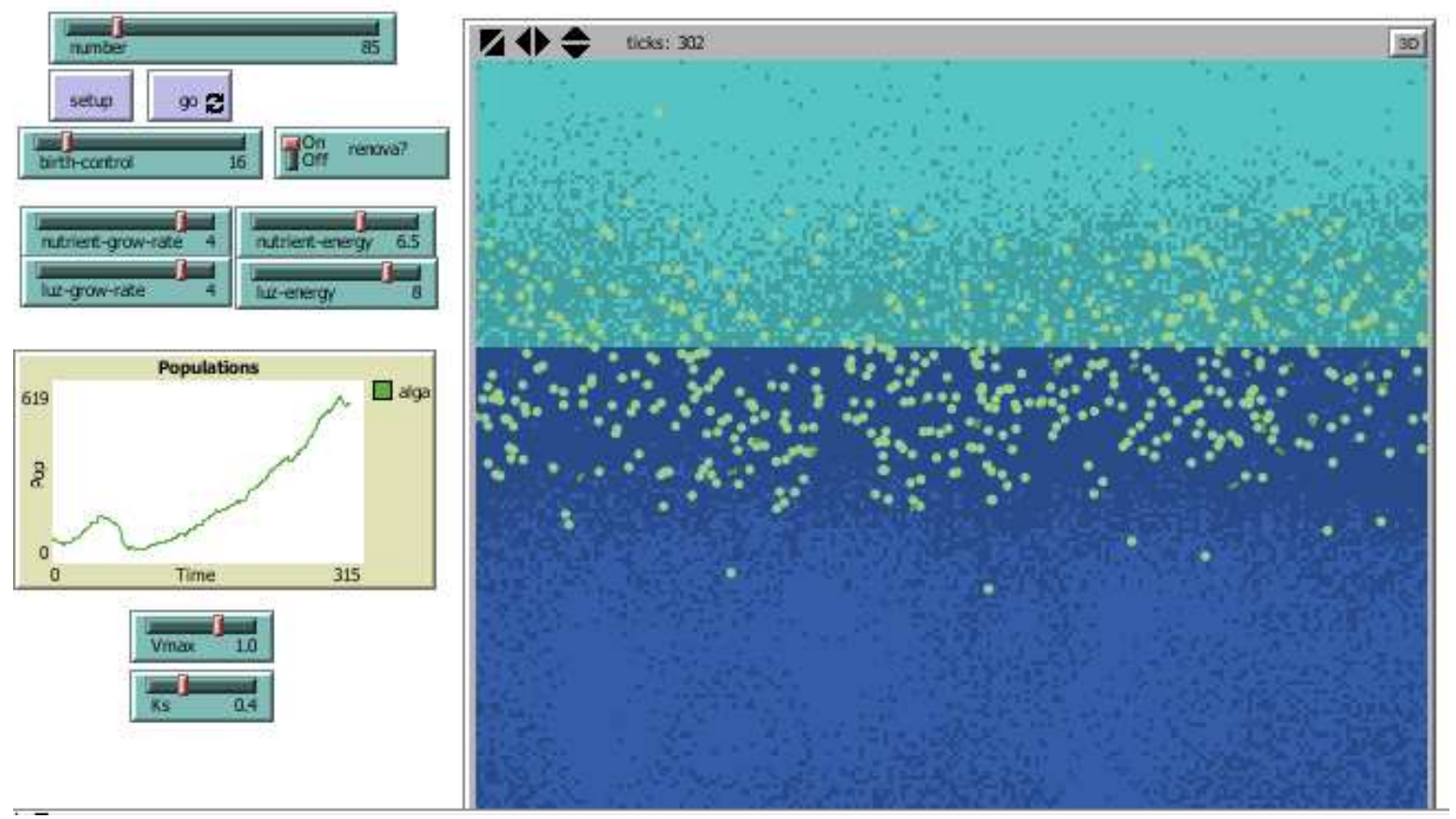


Figura 5: Exemplo de simulação no NetLogo: Funcionamento do Ecossistema Microalgas/Luz/Nutrientes

\section{Conclusões}

A Simulação Baseada em Multiagentes é uma área extremamente interdisciplinar, podendo ser aplicada a diversos domínios. A utilização de uma ferramenta de simulação baseada em agentes torna o processo de aprendizagem facilitado nesta área. Existem diversas ferramentas disponíveis, como Cormas (2011), SWARM (Daniels, 2011) e Madkit (Gutknecht e Ferber, 2011).

A escolha das ferramentas SIMULA(Frozza, 1997) e NetLogo (NetLogo, 2011) devemse ao fato de serem extremamente simples de utilizar.

No caso do SIMULA, a ferramenta apresenta diversas restrições, como quantidade de agentes, tamanho do ambiente (grid) e definição de regras de movimentação (rotinas pré-definidas). Essa ferramenta trabalha apenas com agentes reativos e não tem suporte a comunicação. Sua grande vantagem é a implementação em Java, permitindo utilização em diferentes Sistemas Operacionais.

No caso do NetLogo, a maior restrição está na linguagem utilizada para implementação, o LOGO. Contudo, a ferramenta é extremamente completa, permitindo grande número de agentes (milhares), movimentações, comunicação e uso de agentes reativos e cognitivos. A criação de interface gráfica é facilitada, permitindo inicialização de variáveis, definição de gráficos que apresentam dados em tempo real, etc. Além disso, é uma ferramenta muito estável.

Para as atividades de graduação e pós-graduação propostas, onde os alunos foram introduzidos a área de agentes, iniciando com a utilização do ambiente SIMULA, que atende todos os requisitos para simulação baseada em multiagentes, mas que é mais simples. Em seguida, foram introduzidos a um ambiente mais complexo (relacionado a quantidade de procedimentos possíveis), o NetLogo. Dessa metodologia auxiliou na formação dos alunos na área de agentes e simulação baseada em multiagentes, correspondendo amplamente aos objetivos propostos. Aos alunos de graduação foi dado um tempo maior para desenvolvimento das atividades propostas. Contudo, tanto os alunos de graduação quanto os de pós-graduação apresentaram respostas a essas atividades de forma muito produtiva, visto a escolha dos temas e o nível dos trabalhos desenvolvidos.

Para execução dos trabalhos, os alunos foram instigados a estudar e pensar "como" os problemas escolhidos funcionavam, de forma a realizar a modelagem e posteriormente a implementação das simulações. Interessante perceber que todas as escolhas de problemas foram de áreas não-exatas, como fisiologia, biologia e jogos, fazendo com a interdisciplinariedade fosse colocada em prática. Assim, do ponto de vista didático, os resultados do trabalho proposto foram completamente positivos, pois os alunos aprenderam uma nova técnica computacional e a aplicaram de maneira efetiva.

Como trabalhos futuros, espera-se realizar novos experimentos, fazendo uma análise de como os problemas apresentados podem ser solucionados sem a utilização de simulação baseada em agentes, e se a técnica, de fato, facilita no processo como um todo. 


\section{Agradecimentos}

Gostaria de agradecer aos alunos do 4o. ano de Engenharia de Computacao da FURG da disciplina de Sistemas Inteligentes, nos anos de 2009 e 2010, bem como os alunos do Mestrado em Modelagem Computacional da disciplina de Simulacao Social do ano de 2010.

\section{Referências Bibliográficas}

ALVARES, L. O. C.; SICHMAN, J. S. Introdução aos sistemas multiagentes. In: Jornada de Atualização em Informática, pag 1-37, Brasília - UnB, 1997. Sociedade Brasileira de Computação

CARLOS, J. G. Interdisciplinaridade no ensino médio: desafios e potencialidades. http://vsites.unb.br/ppgec/dissertacoes/proposicoes/proposicao jairocarlos.pdf. Acesso em: 01 de Jun. 2011.

CORMAS. Natural resources and multi-agent simulations, 2011. http://cormas.cirad.fr. Acesso em: 01 de Jun. 2011.

DANIELS, M. The swarm simulation system - a tool for studying complex systems, 2011. <www.swarm.org>. Acesso em: 01 de Jun. 2011.

DROGOUL, A.; FERBER, J. Multi-agent simulation as a tool for modeling societies: Application to social differentiation in ant colonies. In: Proceedings of MAAMAW'92, Viterbo, 1992. Elsevier North-Holland.

FAZENDA, I.C.A. Interdisciplinaridade: História, teoria e pesquisa. Papirus Editora, São Paulo, 1994.

FROZZA, R. SIMULA: Ambiente para desenvolvimento de sistemas multiagentes reativos. Dissertação de mestrado, UFRGS, Universidade Federal do Rio Grande do Sul - Brasil, Rio Grande do Sul, Brasil, 1997.

GILBERT, N.; TROITZCH, K. G. Simulation for the Social Scientist. Buckingham and Philadelphi: Open University Press, London, 1999.

GUTKNECHT, O.; FERBER, J. The madkit project: a multi-agent development kit, 2011. <http://www.madkit.org/>. Acesso em: 01 de Jun. 2011.

NetLogo. http://ccl.northwestern.edu/netlogo/, 2011. Acesso em: 01 de Jun. 2011.

REBONATTO, M. T. Um estudo sobre simulacao paralela. Master's thesis, Instituto de Informática, UFRGS, Porto Alegre, 1999. Mestrado em Ciência da Computação.

STRACK, J. GPSS: modelagem e simulacao de sistemas. LTC, Rio de Janeiro, 1984. $174 \mathrm{p}$. 